\title{
FRACTURA RADICULAR HORIZONTAL. SEGUIMIENTO DE 22 MESES.
}

Juan Carlos León Garzón, OD , Residente II año Edodoncia USTA

Autor responsable de correspondencia:Juan Carlos León Garzón

E-mail: jcleong@ustabuca.edu.co

\begin{abstract}
RESUMEN
La fractura radicular horizontal está asociada con un bajo porcentaje de todos los eventos de trauma dentoalveolar, recibidos en nuestra consulta. Pero lo que agobia dia a dia es que, cuando esto se presenta, la gran interrogante es, ¿qué éxito tendrá esta condición?, y ¿cómo se afectará sicológicamente el paciente, cuando aún mantiene un diente traumatizado en la boca, que probablemente pueda perder?

El objetivo de este artículo es demostrar que un caso que se daba por perdido, debido a un tratamiento odontológico, que desmejoró el pronóstico, salió adelante, con tan solo realizar el retratamiento endodóntico y el control casual después de 22 meses, cuando el paciente volvió a consulta, requiriendo servicio para otra patología en otra estructura dental.[León JC. Fractura Radicular Horizontal. Seguimiento de 22 meses. Ustasalud 2003;2:47-49]
\end{abstract}

PALABRAS CLAVE: Fractura radicular horizontal, Caso clínico, Reabsorción.

Horizontal Root Fracture: 22 Month Follow-up.

\begin{abstract}
Horizontal root fracture is associated to a low percent of all events of dental trauma that come in the dental office. But day to day, this is oppress, when it is present, the big questions are: ¿Will be successful this case? ¿What is the mental result on this patient? ¿How long will be the tooth in the mouth? Finally, after all type of efforts, the patient could loose the tooth.
\end{abstract}

The purpose of this case report is to demonstrate how's a loss case, by a dental treatment with a low pronostique, was success

KEY WORDS: Horizontal root fracture, Report of a case, Reabsortion.

Recibido para publicación: 10 abril de 2003, Aprobado para publicación: Mayo 5 de 2003

\section{INTRODUCCIÓN}

Las fracturas radiculares representan entre un 3 y $5 \%$, de todos los eventos de trauma dentoalveolar. ${ }^{1}$ Una vez diagnosticado, el objetivo fundamental es realizar un tratamiento con la respectiva inducción de cicatrización entre los segmentos sobre las fracturas radiculares.

Andreasen y Hjortin-Hansen ${ }^{2}$, determinaron cuatro tipos de reparación:

Tipo 1: Cicatrización con tejido calcificado.

Tipo 2: Cicatrización con tejido conectivo interproximal.
Tipo 3: Cicatrización con hueso y tejido conectivo interproximal

Tipo 4: Tejido de granulación interproximal sin cicatrización.

Sin embargo, la reparación es el resultado final que se ha relacionado con otras variables, objeto de evaluación, en la primera cita. 
La condición psicológica del paciente es de gran estímulo para el tratamiento, la explicación del proceso, el examen clínico, las ayudas radiográficas, la reposición, la fijación de la estructura dental afectada, el tiempo transcurrido desde el momento del trauma dentoalvelar, la formación apical, la movilidad, el tercio apical comprometido, son algunos, si no todos, los puntos por considerar.

Buralli y Alibrand ${ }^{3}$ a su vez, incluyen dos factores muy importantes en la decisión clínica; las denominan por clasificación de la etiología de las fracturas radiculares: Grupo 1: Dientes no tratados endodónticamente.

Grupo 2: Dientes tratados endodónticamente.

Se debe tener en cuenta adicionalmente, que una baja colaboración por parte del paciente sumado a un control inadecuado, podrían conducir a la pérdida de la estructura afectada.

\section{CASO CLÍNICO}

En Junio 8, de 2001, llegó a consulta un individuo de género masculino y 19 años de edad, que registró dolor a la percusión sobre el incisivo central superior derecho (11), al cual ya se había realizado el tratamiento de conducto según lo manifestó en sus palabras.

Al hacer el examen clínico se encontró que había pigmentación coronal e inflamación sobre la superficie vestibular, que reflejaba dolor en la zona de dientes ánterosuperiores, leve movilidad, no habia fístula y tampoco se encontró bolsa periodontal. Se le interrogó por antecedentes de trauma dentoalveolar, $\mathrm{y}$, el paciente manifestó haber recibido un golpe 6 meses antes.

La radiografía periapical mostró fractura radicular de tipo horizontal en el tercio medio radicular, con imagen radiopaca intraconducto, correspondiente a un proceso endodóntico realizado 3 meses antes, que presentaba pobres condiciones de preparación y obturación. Periapicalmente se evidenciaba una imagen radiolúcida mayor a un centímetro de diámetro, que

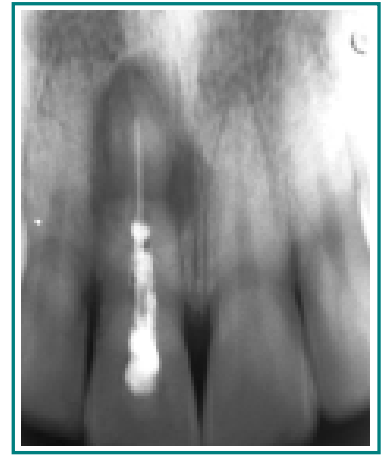

Figura 1. Radiografía inicial involucraba el segmento apical fracturado y reabsorción radicular en la zona de unión de los segmentos radiculares (Figura 1).

Se le explicó el estado en que se encontraba el diente y que el pronóstico era desfavorable, que se intentaría un retratamiento, pero que muy probablemente había que intentar una acción quirúrgica. El paciente aceptó la explicación, pero ante todo se evidenciaba su angustia por temor de perder el diente.

Se procedió a infiltrar solución anestésica de lidocaína al $2 \%$ con epinefrina al 1:80000, aislamiento del campo operatorio y apertura cameral palatina con fresa redonda de alta velocidad; se retiró la gutapercha con xilol y se irrigó abundantemente con hipoclorito de sodio al 5,25\%. La longitud de trabajo se tomó con una lima 35 a 26.5 mm (Figura 2).

Se corrigió la longitud debido al traspaso apical a 25mm con lima \# 35 (Figura 3) y se secó el conducto. Posteriormente se le aplicó hidróxido de calcio puro al conducto, se dejó una mota de algodón y se selló temporalmente (Figura 4).

Al paciente se le formuló Amoxicilina, cápsula de 500 mg cada 8 horas durante 7 días, y Rofecoxib, tabletas de $12,5 \mathrm{mg}$ cada 12 horas por 3 días. Se citó una semana después y se le indicó que ante cualquier circunstancia regresara lo más pronto posible.

En junio 15 de 2001, se realizó la segunda sesión; el paciente refirió la eliminación del dolor; ya no había edema de la zona anterosuperior. En

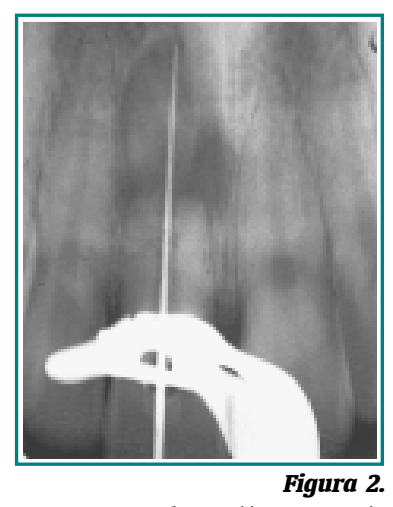

Desobturación y patencia

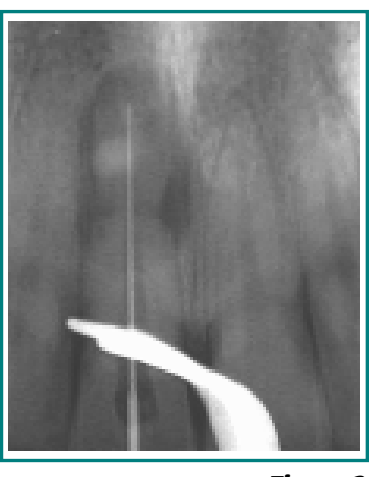

Figura 3. Longitud de trabajo (odontometría)

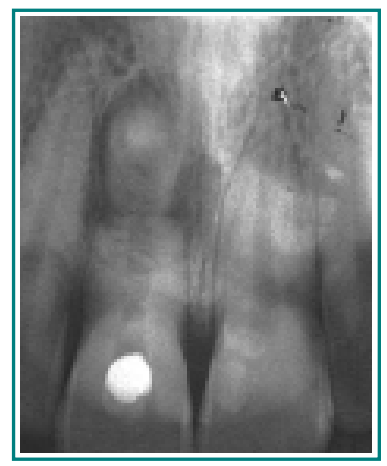

Figura 4. Hidróxido de calcio y selle temporal

48 
esta cita se anestesió nuevamente, aisló y retiró la obturación temporal y el algodón, se removió el hidróxido de calcio y se preparó el conducto hasta una lima 80 a $25 \mathrm{~mm}$ de longitud; se aplicó nuevamente hidróxido de calcio, y se dejó una mota de algodón y obturación temporal. No se siguió ninguna terapia medicamentosa y se citó para los 8 días siguientes

En junio 22 de 2001 regresa el paciente y se realizó la conometría con cono 80 a 25 mm (Figura 5) y se obturó el conducto usando comocementantela pasta de Grossman (laboratorio Eufar-Colombia), y condensación lateral; se aplicó gutapercha blanca y se obturó temporalmente (Figura 6).

El primero de julio, de 2001, se realizó la obturación palatina con resina de auto polimerización TPH color A3. Se dieron las indicaciones acerca de los controles periódicos y se citó a los 3 meses, sin embargo, el paciente nunca volvió.

El 13 de febrero de 2003 el paciente regresó a consulta, porque requería la atención de una obturación coronal sobre el incisivo central superior izquierdo (21). Se tomó una radiografía de control del 11 y se observó total cicatrización periapical de la zona, el paciente manifestó no haber tenido ninguna sintomatología posterior al retratamiento endodóntico (Figura 7).

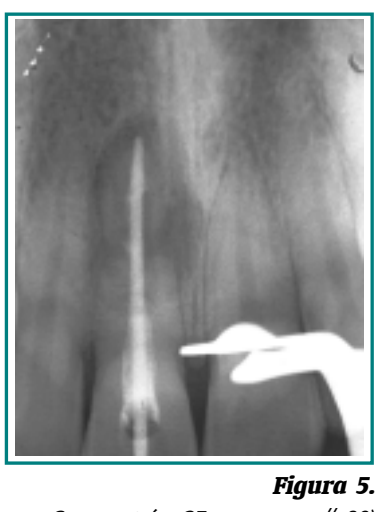

Conometría. 25 mm cono \# 80)

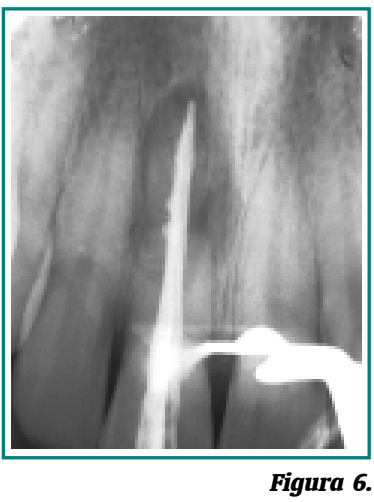

Obturación definitiva

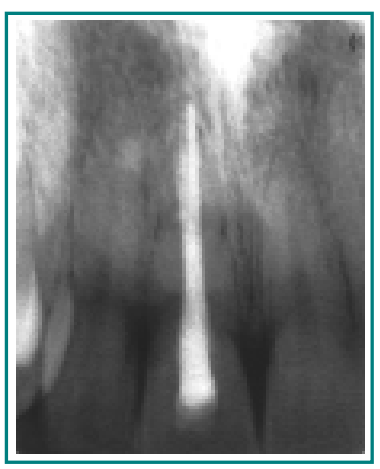

Figura 7.

Radiografia de control a los 2 meses

\section{DISCUSIÓN}

En estos casos por lo general, el pronóstico es demasiado pobre, debido al tiempo transcurrido después del trauma dentoalveolar, sumado a un procedimiento odontológico mal desarrollado, factores que en conjunto disminuyen ostensiblemente los resultados clínicos.

La reposición y ferulización son los procedimientos mandatorios en todo evento de fractura radicular. Sin embargo, en ciertos tratamientos son contraproducentes por la inducción de reabsorción radicular cuando los controles no son apropiados. Muchas veces, por el tiempo transcurrido entre el evento y la primera atención odontológica no se recomienda por la formación de tejido, entre los segmentos radiculares (tejido calcificado) ${ }^{3-6}$

Si bien es cierto que la cirugía es un procedimiento que corrige prontamente el defecto periapical, siempre hay que pensar en el tratamiento o retratamiento endodóntic $0^{6}$, y agotar hasta el último recurso para mantener el segmento apical, y así mejorar el soporte intraóseo.

En conclusión, la fractura radicular, de tipo horizontal, es uno de los diagnósticos, dentro de la variedad del trauma dentoalveolar, que exige un conocimiento apropiado, que lo hace dinámico en el momento que se presenta. Dos de los factores más importantes son la decisión clínica y la colaboración del paciente, pues ambos juegan un papel importante en el éxito terapéutico.

\section{BIBLIOGRAFÍA}

1. Ingle JI, Bakland LK. Endodontics. 4th ed. Baltimore: Williams \& Wilkins, 1994:764-814.

2. Andreasen FM, Andreasen JO, Bayer T. Prognosis of root-fractured permanent incisosrs prediction of healing modalities. Endod Dent Traumatol 1989; 5: 11-22.

3. Borelli P, Pasquale A. Case report. Unusual horizontal and vertical root Fractures of Maxillary Molars: An 11 Year Follow-up. J 0 E. 1999;25: 136-139.

4. Tsukiboshi M. Treatment Planning for Traumatized Teeth. Japón: Quintessence Publishing 2000. p.47-57.

5. Oikarinem $\mathrm{K}$. Tooth splinting: a review of the literature and consideration of the versatility of a wire-composite splint. Endod Dent Traumatol 1.990; 6: 237-250. 